\title{
Infinite-Dimensionality modulo Absolute Borel Classes
}

\author{
by \\ Vitalij CHATYRKO and Yasunao HATTORI \\ Presented by Czestaw BESSAGA \\ Dedicated to Professor Tetsuo Furumochi on his sixtieth birthday \\ Summary. For each ordinal $1 \leq \alpha<\omega_{1}$ we present separable metrizable spaces $X_{\alpha}, Y_{\alpha}$ \\ (i) $\mathrm{f} X_{\alpha}, \mathrm{f} Y_{\alpha}, \mathrm{f} Z_{\alpha}=\omega_{0}$, where $\mathrm{f}$ is either trdef or $\mathcal{K}_{0}$-trsur, \\ (ii) $A(\alpha)$-trind $X_{\alpha}=\infty$ and $M(\alpha)$-trind $X_{\alpha}=-1$, \\ (iii) $A(\alpha)$-trind $Y_{\alpha}=-1$ and $M(\alpha)$-trind $Y_{\alpha}=\infty$, and \\ (iv) $A(\alpha)$-trind $Z_{\alpha}=M(\alpha)$-trind $Z_{\alpha}=\infty$ and $A(\alpha+1) \cap M(\alpha+1)$-trind $Z_{\alpha}=-1$.
} and $Z_{\alpha}$ such that

We also show that there exists no separable metrizable space $W_{\alpha}$ with $A(\alpha)$-trind $W_{\alpha} \neq \infty$, $M(\alpha)$-trind $W_{\alpha} \neq \infty$ and $A(\alpha) \cap M(\alpha)$-trind $W_{\alpha}=\infty$, where $A(\alpha)$ (resp. $\left.M(\alpha)\right)$ is the absolutely additive (resp. multiplicative) Borel class.

1. Introduction. All topological spaces in this paper are assumed to be separable metrizable, and all classes of topological spaces are assumed to be non-empty (the empty space $\emptyset$ is a member of each class), and to contain every space homeomorphic to a closed subspace of each of their members (one says that the class is monotone with respect to closed subspaces). The letter $\mathcal{P}$ is used to denote such a class. Our terminology mostly follows [1] and [3].

In [4] Lelek introduced the small inductive dimension modulo a class $\mathcal{P}$, $\mathcal{P}$-ind, a natural generalization of the small inductive dimension ind and the small inductive compactness degree cmp. Namely, for a space $X$ one defines

2000 Mathematics Subject Classification: Primary 54F45; Secondary 04A15, 54D35, $54 \mathrm{H} 05$.

Key words and phrases: small transfinite inductive dimension modulo $\mathcal{P}$, separable metrizable space, absolute Borel class.

The second author was partially supported by Grant-in-Aid for Scientific Research (No. 19540086) from the Japan Society for Promotion of Science. 
(i) $\mathcal{P}$-ind $X=-1$ iff $X \in \mathcal{P}$,

(ii) $\mathcal{P}$-ind $X \leq n$, where $n$ is an integer $\geq 0$, if for each point $x \in X$ and each closed subset $A$ of $X$ with $x \notin A$ there exists a partition $C$ in $X$ between $x$ and $A$ such that $\mathcal{P}$-ind $C<n$.

Recall that a subset $C$ of a space $X$ is said to be a partition between disjoint sets $A$ and $B$ in $X$ if there are disjoint open subsets $U$ and $V$ of $X$ such that $A \subset U, B \subset V$ and $C=X \backslash(U \cup V)$.

It is evident that if $\mathcal{P}=\{\emptyset\}$ (resp. $\mathcal{P}$ is the class of compact spaces) then $\mathcal{P}$-ind $X=$ ind $X$ (resp. $\mathcal{P}$-ind $X=\operatorname{cmp} X$ ). Moreover, if $\mathcal{P}_{2} \subset \mathcal{P}_{1}$ then $\mathcal{P}_{1}$-ind $X \leq \mathcal{P}_{2}$-ind $X$. In particular, $\operatorname{cmp} X \leq$ ind $X$.

Recall (cf. [1]) that the absolutely additive (resp. multiplicative and ambiguous) Borel classes $A(0), \ldots, A(\alpha), \ldots$ (resp. $M(0), \ldots, M(\alpha), \ldots$ and $A(0) \cap M(0), \ldots, A(\alpha) \cap M(\alpha), \ldots)$, where $0 \leq \alpha<\omega_{1}$, satisfy the conditions above (see Section 3 for details). In the universe of separable metrizable spaces, $A(0)=\{\emptyset\}, M(0)$ is the class $\mathcal{K}_{0}$ of compact metrizable spaces, $A(1)$ is the class $\mathcal{S}_{0}$ of $\sigma$-compact separable metrizable spaces, and $M(1)$ is the class $\mathcal{C}_{0}$ of separable completely metrizable spaces (cf. [1]).

Moreover, the following hierarchy of absolute Borel classes holds (the arrows indicate inclusions of classes):

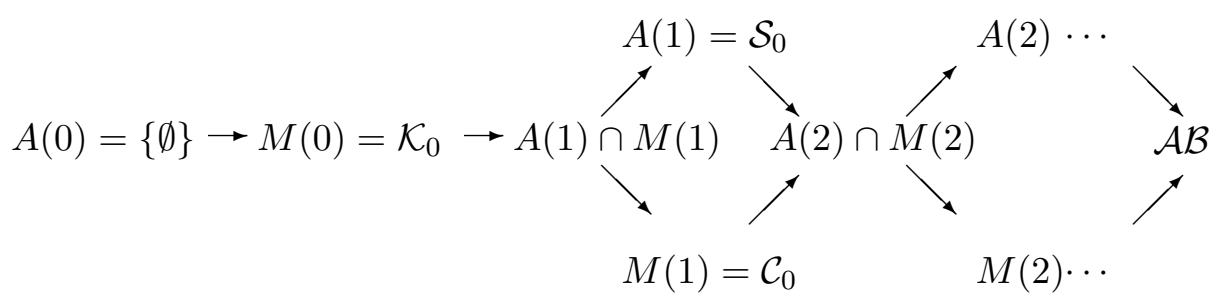

where $\mathcal{A B}=\bigcup\left\{A(\alpha): \alpha<\omega_{1}\right\}$.

It is well known that the Hilbert cube $\mathbb{I}^{\infty}$ has trind $\mathbb{I}^{\infty}=\infty$, where trind is the small transfinite inductive dimension, a natural transfinite extension of ind. Evidently, trcmp $\mathbb{I}^{\infty}=-1$. Hence from the hierarchy it follows that all other small transfinite dimensions of $\mathbb{I}^{\infty}$ modulo absolute Borel classes $\mathcal{P} \neq\{\emptyset\}$ are equal to -1 .

In [5] E. Pol defined the small transfinite inductive compactness degree, trcmp, a natural transfinite extension of $\mathrm{cmp}$, and constructed a separable completely metrizable $\sigma$-compact space $E$ such that $\operatorname{trcmp} E=\infty$. Note that $A(1) \cap M(1)$-trind $E=-1$. Hence by the hierarchy we have trind $E=\infty$, and all other small transfinite dimensions of $E$ modulo absolute Borel classes $\mathcal{P} \supset \mathcal{K}_{0}$ are equal to -1 .

In [2] Charalambous suggested considering a natural transfinite extension of $\mathcal{P}$-ind, $\mathcal{P}$-trind, a generalization of both trind and trcmp (see Section 2 
for the definition). Now the problem naturally arises about analogs of the spaces $\mathbb{I}^{\infty}$ and $E$ for all small transfinite dimensions modulo absolute Borel classes different from trind and trcmp.

Recall that a space $Y$ is a $\mathcal{P}$-hull (resp. a $\mathcal{P}$-kernel) of a space $X$ if $X \subset Y$ (resp. $Y \subset X$ ) and $Y \in \mathcal{P}$. As in [2] the small transfinite $\mathcal{P}$-deficiency and the small transfinite $\mathcal{P}$-surplus of a space $X$ are defined by

$$
\begin{aligned}
& \mathcal{P} \text {-trdef } X=\min \{\operatorname{trind}(Y \backslash X): Y \text { is a } \mathcal{P} \text {-hull of } X\}, \\
& \mathcal{P} \text {-trsur } X=\min \{\operatorname{trind}(X \backslash Y): Y \text { is a } \mathcal{P} \text {-kernel of } X\},
\end{aligned}
$$

respectively. Evidently, the functions $\mathcal{P}$-trdef and $\mathcal{P}$-trsur are transfinite extensions of the functions $\mathcal{P}$-def and $\mathcal{P}$-sur from [1]. Observe (cf. [1]) that for $\mathcal{P}=\mathcal{K}_{0}$ the function $\mathcal{P}$-def is the compact deficiency, def. We will denote the transfinite extension $\mathcal{K}_{0}$-trdef of def by trdef. Note that if $\mathcal{P}_{2} \subset \mathcal{P}_{1}$ then $\mathcal{P}_{1}$-trdef $X \leq \mathcal{P}_{2}$-trdef $X$ and $\mathcal{P}_{1}$-trsur $X \leq \mathcal{P}_{2}$-trsur $X$.

Using an idea of E. Pol, Charalambous [2] presented a space $C$ such that $\mathcal{C}_{0}$-trdef $C=\omega_{0}$ and $\mathcal{C}_{0}$-trind $C=\infty$. This example showed that the Aarts equality $\mathcal{C}_{0}$-def $X=\mathcal{C}_{0}$-ind $X$ (valid for each space $X$ ) cannot be extended to the transfinite case. Recall (cf. [1]) the equalities $M(\alpha)$-def $X=M(\alpha)$-ind $X$ and $A(\alpha)$-sur $X=A(\alpha)$-ind $X$, which hold for each ordinal $1 \leq \alpha<\omega_{1}$ and each space $X$. So the problem arises about extending Charalambous' result to all absolute Borel classes.

The main result of this paper answers the above problems as follows.

TheOREm 1.1. For each ordinal $1 \leq \alpha<\omega_{1}$ there exist spaces $X_{\alpha}, Y_{\alpha}$, $Z_{\alpha}$ such that

(i) f $X_{\alpha}$, f $Y_{\alpha}$, f $Z_{\alpha}=\omega_{0}$, where f is either trdef or $\mathcal{K}_{0}$-trsur,

(ii) $A(\alpha)$-trind $X_{\alpha}=\infty$ and $M(\alpha)$-trind $X_{\alpha}=-1$,

(iii) $A(\alpha)$-trind $Y_{\alpha}=-1$ and $M(\alpha)$-trind $Y_{\alpha}=\infty$,

(iv) $A(\alpha)$-trind $Z_{\alpha}=M(\alpha)$-trind $Z_{\alpha}=\infty$ and $A(\alpha+1) \cap M(\alpha+1)$ trind $Z_{\alpha}=-1$,

but there exists no space $W_{\alpha}$ such that

$$
\begin{array}{r}
A(\alpha) \text {-trind } W_{\alpha} \neq \infty, \\
M(\alpha) \text {-trind } W_{\alpha} \neq \infty, \\
A(\alpha) \cap M(\alpha) \text {-trind } W_{\alpha}=\infty .
\end{array}
$$

Remark 1.1. For each space $X$ and each $1 \leq \alpha<\omega_{1}$ we have:

(i) if $M(\alpha)$-trdef $X \leq \omega_{0}$ and $M(\alpha)$-trind $X=\infty$ (resp. -1 ), then $M(\alpha)$-trdef $X=\omega_{0}$ (resp. -1$)$,

(ii) if $A(\alpha)$ - $\operatorname{trsur} X \leq \omega_{0}$ and $A(\alpha)$-trind $X=\infty$ (resp. -1 ), then $A(\alpha)$ $\operatorname{trsur} X=\omega_{0}($ resp. -1$)$. 
Hence we know additionally that $M(\alpha)$-trdef $X_{\alpha}=A(\alpha)$-trsur $Y_{\alpha}=-1$ and $A(\alpha)-\operatorname{trsur} X_{\alpha}=M(\alpha)-\operatorname{trdef} Y_{\alpha}=M(\alpha)-\operatorname{trdef} Z_{\alpha}=A(\alpha)-\operatorname{trsur} Z_{\alpha}=\omega_{0}$.

Theorem 1.2. There exists a space $X$ with $\operatorname{trdef} X=\mathcal{K}_{0}$-trsur $X=\omega_{0}$ such that $\mathcal{A B}$-trind $X=\infty$.

REMARK 1.2. By the hierarchy we have B-trind $X=\infty$ for each absolute Borel class B and D-trdef $X=\mathrm{D}$-trsur $X=\omega_{0}$ for each absolute Borel class D except $A(0)$.

2. Small transfinite inductive dimension modulo a class $\mathcal{P}$. Let $X$ be a space and $\alpha$ be either an ordinal $\geq 0$ or the integer -1 .

Recall ([2]) that the small transfinite inductive dimension modulo a class $\mathcal{P}, \mathcal{P}$-trind, of $X$ is defined as follows:

(i) $\mathcal{P}$-trind $X=-1$ iff $X \in \mathcal{P}$.

(ii) $\mathcal{P}$-trind $X \leq \alpha$ ( $\geq 0)$ if for every point $x \in X$ and every closed subset $A$ of $X$ such that $x \notin A$ there exists a partition $C$ in $X$ between $x$ and $A$ with $\mathcal{P}$-trind $C<\alpha$.

(iii) $\mathcal{P}$-trind $X=\alpha$ if $\mathcal{P}$-trind $X \leq \alpha$ and $\mathcal{P}$-trind $X>\beta$ for every ordinal $\beta<\alpha$.

(iv) $\mathcal{P}$-trind $X=\infty$ if $\mathcal{P}$-trind $X>\alpha$ for every ordinal $\alpha$.

Note that $\{\emptyset\}$-trind $=$ trind and $\mathcal{K}_{0}$-trind $=$ trcmp. Some other known functions are $\mathcal{S}_{0}$-trind $=\mathcal{S}$-trind, the small transfinite inductive $\sigma$-compactness degree, and $\mathcal{C}_{0}$-trind $=$ tricd, the small transfinite inductive completeness degree ([2]). The following relationships between particular cases of $\mathcal{P}$-trind are evident.

Proposition 2.1.

(i) $\mathcal{P}_{1}$-trind $=\mathcal{P}_{2}$-trind iff $\mathcal{P}_{1}=\mathcal{P}_{2}$.

(ii) If $\mathcal{P}_{2} \subset \mathcal{P}_{1}$ then $\mathcal{P}_{1}$-trind $\leq \mathcal{P}_{2}$-trind.

(iii) If $X \in \mathcal{P}$ then $\mathcal{P}_{1}$-trind $X=\mathcal{P} \cap \mathcal{P}_{1}$-trind $X$ for every class $\mathcal{P}_{1}$.

Note that trind $\geq \mathcal{P}$-trind for every class $\mathcal{P}$, and trcmp $\geq \max \{\mathcal{S}$-trind, tricd $\}$. Observe also that the function $\mathcal{P}$-trind is monotone with respect to closed subsets.

3. Absolute Borel classes. Recall that every ordinal $\alpha$ can be represented as $\alpha=\lambda(\alpha)+n(\alpha)$, where $\lambda(\alpha)$ is a limit ordinal or 0 , and $n(\alpha)$ is an integer $\geq 0$. An ordinal $\alpha$ is called even (resp. odd) if $n(\alpha)$ is even (resp. odd). As in [1] let us denote by $\mathcal{A}_{\sigma}$ (resp. $\mathcal{A}_{\delta}$ ) the family of all countable unions (resp. intersections) of elements from a family $\mathcal{A}$ of sets.

Let $\mathcal{B}(X)$ be the family of Borel subsets of a space $X$. This family can be generated by an inductive transfinite process (cf. [1]). For each countable 
ordinal $\alpha \geq 0$ the Borel class $F_{\alpha}(X)$ (resp. $G_{\alpha}(X)$ ) is defined transfinitely as follows.

(i) $F_{0}(X)$ (resp. $\left.G_{0}(X)\right)$ is the family of all closed (resp. open) subsets of $X$;

(ii) if $\alpha$ is odd then $F_{\alpha}(X)=\left(\bigcup\left\{F_{\beta}(X): \beta<\alpha\right\}\right)_{\sigma}$ (resp. $G_{\alpha}(X)=$ $\left.\left(\bigcup\left\{G_{\beta}(X): \beta<\alpha\right\}\right)_{\delta}\right)$;

(iii) if $\alpha$ is even then $F_{\alpha}(X)=\left(\bigcup\left\{F_{\beta}(X): \beta<\alpha\right\}\right)_{\delta}\left(\right.$ resp. $G_{\alpha}(X)=$ $\left.\left(\bigcup\left\{G_{\beta}(X): \beta<\alpha\right\}\right)_{\sigma}\right)$.

Notice that $\mathcal{B}(X)=\bigcup\left\{F_{\alpha}(X): \alpha<\omega_{1}\right\}=\bigcup\left\{G_{\alpha}(X): \alpha<\omega_{1}\right\}$. It is also clear that $A \in F_{\alpha}(X)$ iff $X \backslash A \in G_{\alpha}(X)$ (briefly, $F_{\alpha}(X)=\neg G_{\alpha}(X)$ ), and if $Z \subset Y \subset X$ then $Z \in F_{\alpha}(Y)$ (resp. $G_{\alpha}(Y)$ ) iff there exists a subset $Z^{\prime} \subset X$ such that $Z=Z^{\prime} \cap Y$ and $Z^{\prime} \in F_{\alpha}(X)$ (resp. $\left.G_{\alpha}(X)\right)$.

Recall that for each ordinal $0 \leq \alpha<\omega_{1}$ the multiplicative (resp. additive) Borel class $\alpha, \Pi_{\alpha}^{0}(X)$ (resp. $\left.\Sigma_{\alpha}^{0}(X)\right)$ of a space $X$ is the family $F_{\alpha}$ for $\alpha$ even (resp. odd) and $G_{\alpha}$ for $\alpha$ odd (resp. even); the ambiguous class $\alpha, \Delta_{\alpha}^{0}(X)$, of $X$ is $\Pi_{\alpha}^{0}(X) \cap \Sigma_{\alpha}^{0}(X)$. Some properties of multiplicative, additive and ambiguous classes of a space $X$ can be found in the next statement.

Proposition 3.1 ([8, Proposition 3.6.1]).

(i) The additive (resp. multiplicative) classes are closed under countable unions (resp. intersections).

(ii) The additive, multiplicative and ambiguous classes are closed under finite intersections and finite unions.

(iii) The following hierarchy of Borel sets holds (the arrows indicate inclusions of families):

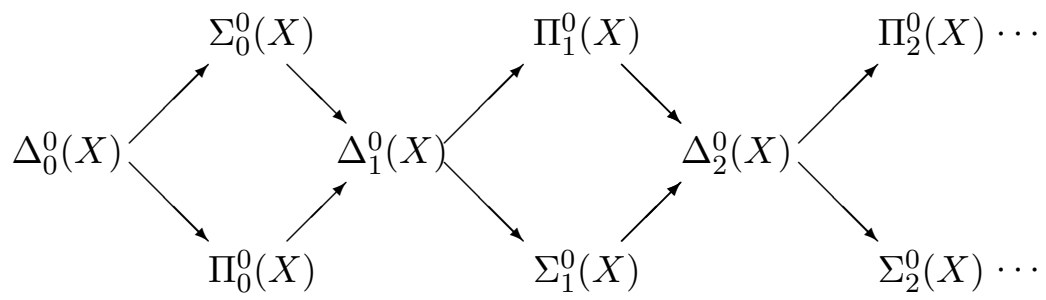

(iv) For each $0 \leq \alpha<\omega_{1}, \Pi_{\alpha}^{0}(X)=\neg \Sigma_{\alpha}^{0}(X)$ and $\Delta_{\alpha}^{0}(X)$ is an algebra.

(v) For each $0<\alpha<\omega_{1}, \Sigma_{\alpha}^{0}(X)=\left(\Delta_{\alpha}^{0}(X)\right)_{\sigma}$ and $\Pi_{\alpha}^{0}(X)=\left(\Delta_{\alpha}^{0}(X)\right)_{\delta}$.

We need two more facts about the Borel sets.

Proposition 3.2 ([8, Corollary 3.6.8]). Let $X$ be an uncountable Polish space and $0 \leq \alpha<\omega_{1}$. Then there exists an element $E$ of $\Sigma_{\alpha}^{0}(X)$ which is not in $\Pi_{\alpha}^{0}(X)$. 
Note that then $X \backslash E \in \Pi_{\alpha}^{0}(X) \backslash \Sigma_{\alpha}^{0}(X)$. Observe that both $E$ and $X \backslash E$ are in $\Delta_{\alpha+1}^{0}(X)$.

Proposition 3.3 ([8, Theorem 5.2.11]). Let $X, Y$ be compact metric spaces and $f: X \rightarrow Y$ a continuous onto mapping. Suppose $A \subset Y$ and $0 \leq \alpha<\omega_{1}$. Then $A \in \Pi_{\alpha}^{0}(Y)$ iff $f^{-1}(A) \in \Pi_{\alpha}^{0}(X)$.

We also notice that if $B \subset Y$ and $0 \leq \alpha<\omega_{1}$ then $B \in \Sigma_{\alpha}^{0}(Y)$ (resp. $\left.\Delta_{\alpha}^{0}(Y)\right)$ iff $f^{-1}(B) \in \Sigma_{\alpha}^{0}(X)\left(\operatorname{resp} . \Delta_{\alpha}^{0}(X)\right)$.

Following [2] we call a subset $A$ of a space $X$ a Bernstein set if $|A \cap B|=$ $|(X \backslash A) \cap B|=c$ (continuum) for every uncountable $B \in \mathcal{B}(X)$. Let $\operatorname{Brn}(X)$ denote the family of all Bernstein sets of $X$. Notice that $\operatorname{Brn}(X) \neq \emptyset$ for every uncountable Polish space $X$. Indeed, recall (cf. [8, Theorem 3.2.7]) that every uncountable Borel subset $B$ of $X$ contains a copy of the Cantor set $C$. Note that $C$ is homeomorphic to $C^{2}$. So $B$ contains $c$ disjoint copies of $C$. One can show as in [8, Example 3.2.8] that $X$ contains a subset $A$ such that $A \cap F$ and $(X \backslash A) \cap F$ are uncountable for each uncountable closed set $F$ in $X$. So $|A \cap B|=|(X \backslash A) \cap B|=c$. Hence $A \in \operatorname{Brn}(X)$.

Note that if $M \in \operatorname{Brn}(X)$ then $|M|=c, X \backslash M \in \operatorname{Brn}(X)$ and $M \notin \mathcal{B}(X)$.

Recall that a space $X$ is said to be absolutely of multiplicative (resp. additive) class $\alpha$, where $0 \leq \alpha<\omega_{1}$, if $X$ is of multiplicative (resp. additive) Borel class $\alpha$ in $Y$ whenever $X$ is a subspace of a space $Y$ (that is, for any homeomorphic embedding $h: X \rightarrow Y$ the image $h(X)$ is of multiplicative (resp. additive) class $\alpha$ in $Y$ ). As in [1] let us denote the absolutely multiplicative (resp. additive) Borel class $\alpha$ by $M(\alpha)$ (resp. $A(\alpha)$ ). For each $0 \leq \alpha<\omega_{1}$ the intersection $M(\alpha) \cap A(\alpha)$ is called the ambiguous absolutely Borel class $\alpha$.

Proposition 3.4 ([1, Theorem II.9.6 and Corollary II.9.7]).

(i) $A(0)=\{\emptyset\}, M(0)=\mathcal{K}_{0}, A(1)=\mathcal{S}_{0}$ and $M(1)=\mathcal{C}_{0}$.

(ii) For every $\alpha$ with $2 \leq \alpha<\omega_{1}$ a space $X$ is in $M(\alpha)$ (resp. $A(\alpha)$ ) iff there is a homeomorphic embedding $h: X \rightarrow Y$ with $Y \in \mathcal{C}_{0}$ such that $h(X)$ is of multiplicative (resp. additive) class $\alpha$ in $Y$.

It is evident that the classes $M(\alpha), A(\alpha)$ and $A(\alpha) \cap M(\alpha), 0 \leq \alpha<\omega_{1}$, are monotone with respect to closed subsets. A class $\mathcal{P}$ of topological spaces is said to be finitely additive if each space $X$, covered by a finite family of elements of $\mathcal{P}$, is also an element of $\mathcal{P}$.

Proposition 3.5 ([1, Theorem II.9.9]). For each ordinal $\alpha<\omega_{1}$ the classes $A(\alpha), M(\alpha)$ and $A(\alpha) \cap M(\alpha)$ are finitely additive.

We will call a space $X$ absolute Borel if $X$ is in $A(\alpha)$ (or $M(\alpha)$ ) for some $\alpha<\omega_{1}$. We denote by $\mathcal{A B}$ the class of all absolute Borel spaces. 
A simple corollary of Propositions 3.1 and 3.4 is the hierarchy of absolute Borel classes in the universe of separable metrizable spaces from the introduction. Let $\mathbb{I}$ denote the closed interval $[0,1], Q_{1}$ the space of rational numbers in $\mathbb{I}$, and $P_{1}$ the space of irrational numbers in $\mathbb{I}$. Observe that $Q_{1} \in \mathcal{S}_{0} \backslash \mathcal{C}_{0}$ and $P_{1} \in \mathcal{C}_{0} \backslash \mathcal{S}_{0}$.

Proposition 3.6. For each ordinal $1 \leq \alpha<\omega_{1}$ there are subsets $Q_{\alpha}, P_{\alpha}$ and $D_{\alpha}$ of $\mathbb{I}$ such that

(i) $Q_{\alpha} \in A(\alpha) \backslash M(\alpha)$ and $P_{\alpha} \in M(\alpha) \backslash A(\alpha)$;

(ii) $D_{\alpha} \in A(\alpha+1) \cap M(\alpha+1)$, but $D_{\alpha} \notin A(\alpha) \cup M(\alpha)$.

Proof. (i) For $\alpha>1$ the desired subsets $Q_{\alpha}$ and $P_{\alpha}$ exist by Propositions 3.2 and 3.4.

(ii) For $\alpha \geq 1$ put $D_{\alpha}=E_{\alpha} \cup F_{\alpha}$, where $E_{\alpha}$ is in $\Sigma_{\alpha}^{0}([0,1 / 3]) \backslash \Pi_{\alpha}^{0}([0,1 / 3])$ and $F_{\alpha}$ is in $\Pi_{\alpha}^{0}([2 / 3,1]) \backslash \Sigma_{\alpha}^{0}([2 / 3,1])$. The statement is proved.

4. Infinite-dimensionality modulo a class $\mathcal{P}$. We will follow some idea of E. Pol from [5].

In this section all classes $\mathcal{P}$ of topological spaces are additionally assumed to be finitely additive. A space $X$ is said to have property $(*)_{\mathcal{P}}$ if for every sequence $\left\{\left(A_{i}, B_{i}\right)\right\}_{i=1}^{\infty}$ of pairs of disjoint compact subsets of $X$ there exist partitions $L_{i}$ between $A_{i}$ and $B_{i}$ such that $\bigcap_{i=1}^{N} L_{i} \in \mathcal{P}$ for some integer $N$. It is evident that if a space $X$ has property $(*)_{\mathcal{P}}$ then so does each closed subset of $X$.

REMARK 4.1. Let $M$ be a subspace of a space $X,(A, B)$ a pair of disjoint closed subsets of $X$, and $L$ a partition in $M$ between $M \cap A$ and $M \cap B$. If $M$ is closed in $X$ or $A, B \subset M$ then there exists a partition $L^{\prime}$ in $X$ between $A$ and $B$ such that $M \cap L^{\prime}=L$ (see [3, Lemma 1.2.9] and [1, Lemma I. 4.5]).

Proposition 4.1. If a space $X$ is covered by a finite family of closed sets, each having property $(*)_{\mathcal{P}}$, then $X$ also has this property.

Proof. It is sufficient to consider the case when $X$ is the union of two closed subsets $X_{1}$ and $X_{2}$ which have property $(*)_{\mathcal{P}}$. Consider a sequence $\left\{\left(A_{i}, B_{i}\right)\right\}_{i=1}^{\infty}$ of pairs of disjoint compact subsets of $X$. Since $X_{1}$ has property $(*)_{\mathcal{P}}$, for each integer $i \geq 0$ there is a partition $L_{2 i+1}^{\prime}$ between $A_{2 i+1} \cap X_{1}$ and $B_{2 i+1} \cap X_{1}$ in $X_{1}$ such that $\bigcap_{i=1}^{N_{1}} L_{2 i+1}^{\prime} \in \mathcal{P}$ for some $N_{1}$. Let $L_{2 i+1}$ be a partition between $A_{2 i+1}$ and $B_{2 i+1}$ in $X$ such that $L_{2 i+1} \cap X_{1}=L_{2 i+1}^{\prime}$. So $\bigcap_{i=1}^{N_{1}}\left(L_{2 i+1} \cap X_{1}\right) \in \mathcal{P}$. Similarly, for each $i \geq 1$ we have a partition $L_{2 i}$ between $A_{2 i}$ and $B_{2 i}$ in $X$ such that $\bigcap_{i=1}^{N_{2}}\left(L_{2 i} \cap X_{2}\right) \in \mathcal{P}$ for some $N_{2}$. Put $N=\max \left\{2 N_{1}+1,2 N_{2}\right\}$. Then 


$$
\begin{aligned}
\bigcap_{i=1}^{N} L_{i} & =\left(\left(\bigcap_{i=1}^{N} L_{i}\right) \cap X_{1}\right) \cup\left(\left(\bigcap_{i=1}^{N} L_{i}\right) \cap X_{2}\right) \\
& =\left(\bigcap_{i=1}^{N}\left(L_{i} \cap X_{1}\right)\right) \cup\left(\bigcap_{i=1}^{N}\left(L_{i} \cap X_{2}\right)\right) \\
& \subset\left(\bigcap_{i=1}^{N_{1}}\left(L_{2 i+1} \cap X_{1}\right)\right) \cup\left(\bigcap_{i=1}^{N_{2}}\left(L_{2 i} \cap X_{2}\right)\right) .
\end{aligned}
$$

Since $\mathcal{P}$ is finitely additive and monotone with respect to closed subsets, we have $\bigcap_{i=1}^{N} L_{i} \in \mathcal{P}$. This completes the proof.

Proposition 4.2. If $\mathcal{P}$-trind $X \neq \infty$ then $X$ has property $(*)_{\mathcal{P}}$.

Proof. Let us apply induction on $\alpha=\mathcal{P}$-trind $X$. If $\alpha=-1$, then $X \in \mathcal{P}$ and the statement is evidently valid.

Assume that the conclusion holds for $\mathcal{P}$-trind $X<\alpha \geq 0$. Let now $X$ have $\mathcal{P}$-trind $X=\alpha$. Consider a sequence $\left\{\left(A_{i}, B_{i}\right)\right\}_{i=1}^{\infty}$ of pairs of disjoint compact subsets of $X$. Since $A_{1}$ is compact and $\mathcal{P}$-trind $X=\alpha$, there exist open subsets $U_{1}, \ldots, U_{k}$ of $X$ such that $A_{1} \subset \bigcup_{i=1}^{k} U_{i}$ and for each $i=$ $1, \ldots, k$, we have $\mathrm{Cl} U_{i} \cap B_{1}=\emptyset$ and $\mathcal{P}$-trind $\mathrm{Bd} U_{i} \leq \beta_{i}$ for some $\beta_{i}<\alpha$. By the inductive assumption and Proposition 4.1, the set $\bigcup_{i=1}^{k} \operatorname{Bd} U_{i}$ has property $(*)_{\mathcal{P}}$. We put $U=\bigcup_{i=1}^{k} U_{i}$. It is easy to see that $L_{1}=\operatorname{Bd} U \subset$ $\bigcup_{i=1}^{k} \mathrm{Bd} U_{i}$ is a partition between $A_{1}$ and $B_{1}$, and $L_{1}$ has property $(*)_{\mathcal{P}}$. Note that for each $i \geq 2$ there exists a partition $L_{i}^{\prime}$ between $A_{i} \cap L_{1}$ and $B_{i} \cap L_{1}$ in $L_{1}$ such that $\bigcap_{i=2}^{N} L_{i}^{\prime} \in \mathcal{P}$ for some $N$. Now if we take, for each $i=2, \ldots, N$, a partition $L_{i}$ between $A_{i}$ and $B_{i}$ in $X$ such that $L_{i} \cap L_{1}=L_{i}^{\prime}$ then $\bigcap_{i=2}^{N} L_{i}^{\prime}=\bigcap_{i=1}^{N} L_{i}$. So $\bigcap_{i=1}^{N} L_{i} \in \mathcal{P}$. The proposition is proved.

Let $\mathbb{I}^{\infty}=\left\{\left(x_{j}\right): 0 \leq x_{j} \leq 1, j=1,2, \ldots\right\}$ be the product of countably many intervals $\mathbb{I}$. For each $n \geq 2$ denote the subset $\left\{\left(x_{j}\right) \in \mathbb{I}^{\infty}: x_{k}=0\right.$ for $k \geq n+1\}$ by $\mathbb{I}^{n}$. For each $n \geq 2$ and each $i=1, \ldots, n$, set $A_{i}^{n}=\left\{\left(x_{j}\right) \in \mathbb{I}^{n}\right.$ : $\left.x_{i}=0\right\}$ and $B_{i}^{n}=\left\{\left(x_{j}\right) \in \mathbb{I}^{n}: x_{i}=1\right\}$. Choose for each $n \geq 2$ a subset $E_{n}$ of $\mathbb{I}^{n}$ and put

$$
X=\left(\{0\} \times \mathbb{I}^{\infty}\right) \cup \bigcup_{n=2}^{\infty}\left(\{1 / n\} \times E_{n}\right) .
$$

Let $Y=\left(\{0\} \times \mathbb{I}^{\infty}\right) \cup \bigcup_{n=2}^{\infty}\left(\{1 / n\} \times \mathbb{I}^{n}\right)$ and $Z=\{0,1 / 2,1 / 3, \ldots\}$. It is obvious that $X \subset Y \subset Z \times \mathbb{I}^{\infty}$. Moreover, $Y$ is compact, and its subspace $Y \backslash X$ is a topological sum of countably many finite-dimensional spaces. Hence, $\operatorname{trind}(Y \backslash X) \leq \omega_{0}$. Moreover, $\operatorname{trind}\left(X \backslash\left(\{0\} \times \mathbb{I}^{\infty}\right)\right) \leq \omega_{0}$. It follows that

$$
\operatorname{trdef} X \leq \omega_{0} \quad \text { and } \quad \mathcal{K}_{0} \text {-trsur } X \leq \omega_{0} .
$$


Proposition 4.3. If for each integer $m \geq 1$ there exist an integer $k(m) \geq m+1$ such that for every $n \geq k(m)$ and for arbitrary partitions $L_{i}^{n}$ between $A_{i}^{n}$ and $B_{i}^{n}$ in $\mathbb{I}^{n}, i \leq n$, we have $E_{n} \cap \bigcap_{i=1}^{m} L_{i}^{n} \notin \mathcal{P}$, then $\mathcal{P}$-trind $X=\infty$.

Proof. We will apply Proposition 4.2. For each $i \geq 1$ let $L_{i}$ be an arbitrary partition between the compact sets $A_{i}=\left\{\left(0,\left(x_{j}\right)\right) \in\{0\} \times \mathbb{I}^{\infty}: x_{i}=0\right\}$ and $B_{i}=\left\{\left(0,\left(x_{j}\right)\right) \in\{0\} \times \mathbb{I}^{\infty}: x_{i}=1\right\}$ in $X$. It suffices to show that $\bigcap_{i=1}^{N} L_{i} \notin \mathcal{P}$ for every $N \geq 1$. Consider, for each $i \geq 1$, a partition $L_{i}^{\prime}$ between $A_{i}$ and $B_{i}$ in $Y$ such that $L_{i}^{\prime} \cap X=L_{i}$. Note that for every $i$ there exists an integer $n_{i} \geq 2$ such that for each $n \geq n_{i}$ the set $L_{i}^{n}=L_{i}^{\prime} \cap\left(\{1 / n\} \times \mathbb{I}^{n}\right)$ is a partition between $\{1 / n\} \times A_{i}^{n}$ and $\{1 / n\} \times B_{i}^{n}$ in $\{1 / n\} \times \mathbb{I}^{n}$. Let $N$ be an arbitrary integer and $n=\max \left\{n_{1}, \ldots, n_{N}, k(N)\right\}$. Note that

$$
\begin{aligned}
C & =\left(\bigcap_{i=1}^{N} L_{i}^{n}\right) \cap\left(\{1 / n\} \times E_{n}\right)=\left(\bigcap_{i=1}^{N} L_{i}^{\prime}\right) \cap\left(\{1 / n\} \times E_{n}\right) \\
& =\left(\bigcap_{i=1}^{N} L_{i}\right) \cap\left(\{1 / n\} \times E_{n}\right)
\end{aligned}
$$

is a closed subset of $\bigcap_{i=1}^{N} L_{i}$. Moreover, $C \notin \mathcal{P}$ by the assumption. Hence $\bigcap_{i=1}^{N} L_{i} \notin \mathcal{P}$. The proposition is proved.

Let us recall the following.

Proposition 4.4 ([7, Lemma 5.2]). Let $L_{i_{j}}, j=1, \ldots, p$, be partitions between the opposite faces $A_{i_{j}}^{n}$ and $B_{i_{j}}^{n}$ in $\mathbb{I}^{n}$, where $1 \leq i_{1}<\cdots<i_{p} \leq n$ and $1 \leq p<n$. Then for any $k \neq i_{j}, j=1, \ldots, p$, there is a continuum $C \subset \bigcap_{j=1}^{p} L_{i_{j}}$ meeting the faces $A_{k}^{n}$ and $B_{k}^{n}$.

Now we are ready to prove

Proposition 4.5. Let $L_{i}$ be a partition between the opposite faces $A_{i}^{n}$ and $B_{i}^{n}$ in the cube $\mathbb{I}^{n}, i \leq p$, for some $p<n$. Let also $L=\bigcap_{i=1}^{p} L_{i}$, $E=\left\{\left(x_{i}\right) \in \mathbb{I}^{n}: x_{n} \in F\right\} \subset \mathbb{I}^{n}$, where $F$ is a subset of $[0,1]$, and let $Q_{\alpha}, P_{\alpha}$, $D_{\alpha}, 1 \leq \alpha<\omega_{1}$, be the subsets of $[0,1]$ from Proposition 3.6. Then

(i) $L \cap E \notin \mathcal{K}_{0}$ if $F \notin \mathcal{K}_{0}$,

(ii) $L \cap E \notin M(\alpha)$ if $F=Q_{\alpha}$,

(iii) $L \cap E \notin A(\alpha)$ if $F=P_{\alpha}$,

(iv) $L \cap E \notin M(\alpha) \cup A(\alpha)$ if $F=D_{\alpha}$,

(v) $L \cap E$ is not a Borel set of $\mathbb{I}^{n}$ if $F \in \operatorname{Brn}([0,1])$.

Proof. By Proposition 4.4 there is a continuum $C \subset L$ meeting the faces $A_{n}^{n}$ and $B_{n}^{n}$. Let $\pi_{n}$ be the projection of $\mathbb{I}^{n}$ onto the $n$th coordinate $\mathbb{I}$, i.e., $\pi_{n}\left(x_{1}, \ldots, x_{n}\right)=x_{n}$, and let $\pi_{n}^{C}$ be the restriction of $\pi_{n}$ to $C$. Observe that 
$\pi_{n}^{C}$ is a continuous mapping of $C$ onto $[0,1]$. Notice also that $C \cap E=$ $\left(\pi_{n}^{C}\right)^{-1}(F)$ and $C \cap E$ is a closed subset of $L \cap E$.

(i) Since $F \notin \mathcal{K}_{0}$ we have $C \cap E=\left(\pi_{n}^{C}\right)^{-1}(F) \notin \mathcal{K}_{0}$, and so $L \cap E \notin \mathcal{K}_{0}$.

(ii) Since $Q_{\alpha} \notin M(\alpha)$, by Proposition 3.3 we have $C \cap E=\left(\pi_{n}^{C}\right)^{-1}\left(Q_{\alpha}\right) \notin$ $M(\alpha)$, and hence $L \cap E \notin M(\alpha)$.

(iii) Since $P_{\alpha} \notin A(\alpha)$, by Proposition 3.3 we have $C \cap E=\left(\pi_{n}^{C}\right)^{-1}\left(P_{\alpha}\right) \notin$ $A(\alpha)$, and hence $L \cap E \notin A(\alpha)$.

(iv) Since $D_{\alpha}$ is neither in $M(\alpha)$ nor in $A(\alpha)$, Proposition 3.3 shows that $C \cap E=\left(\pi_{n}^{C}\right)^{-1}\left(D_{\alpha}\right) \notin M(\alpha) \cup A(\alpha)$, and hence $L \cap E$ has the same property.

(v) Since $F$ is not a Borel set in $[0,1], C \cap E=\left(\pi_{n}^{C}\right)^{-1} F$ is not a Borel set by Proposition 3.3. Hence $L \cap E$ is not a Borel set in $\mathbb{I}^{n}$.

Lemma 4.1. For every space $Y$, if $\operatorname{trdef} Y \leq \omega_{0}$ and $\operatorname{trcmp} Y=\infty$, then $\operatorname{trdef} Y=\omega_{0}$.

Proof. Observe that if $\operatorname{trdef} Y$ is finite so $\operatorname{trdef} Y=\operatorname{def} Y \geq \operatorname{cmp} Y$ (the last inequality can be found in [1]). This contradiction proves the lemma.

Proposition 4.6. Let $F$ be a non-compact subset of $\mathbb{I}$ and $X$ from (4.1), where $E_{n}=\left\{\left(x_{i}\right) \in \mathbb{I}^{n}: x_{n} \in F\right\}$ for each $n \geq 2$. Then $\operatorname{trcmp} X=\infty$ and $\operatorname{trdef} X=\mathcal{K}_{0}$-trsur $X=\omega_{0}$. Moreover, for any $1 \leq \alpha<\omega_{1}$ we have:

(i) if $F=Q_{\alpha}$, then $M(\alpha)$-trind $X=\infty, A(\alpha)$-trind $X=A(\alpha)$-trsur $X$ $=-1$ and $M(\alpha)$-trdef $X=\omega_{0}$,

(ii) if $F=P_{\alpha}$, then $A(\alpha)$-trind $X=\infty, M(\alpha)$-trind $X=M(\alpha)$-trdef $X$ $=-1$ and $A(\alpha)$-trsur $X=\omega_{0}$,

(iii) if $F=D_{\alpha}$, then $M(\alpha)$-trind $X=A(\alpha)$-trind $X=\infty, A(\alpha+1)$ $\cap M(\alpha+1)$-trind $X=-1$ and $M(\alpha)$-trdef $X=M(\alpha)$-trsur $X=\omega_{0}$,

(iv) if $F \in \operatorname{Brn}([0,1])$, then $\mathcal{A B}$-trind $X=\infty$.

Proof. For each integer $m \geq 1$ put $k(m)=m+1$. Consider $m \geq 1$ and $n \geq k(m)$. Let $L_{i}^{n}$ be an arbitrary partition between $A_{i}^{n}$ and $B_{i}^{n}$ in $\mathbb{I}^{n}$ for each $i=1, \ldots, m$. By Proposition 4.5(i) we have $E_{n} \cap \bigcap_{i=1}^{m} L_{i}^{n} \notin \mathcal{K}_{0}$. Hence, by Proposition 4.3, it follows that $\operatorname{trcmp} X=\infty$. Then, by Lemma 4.1 and (4.2), we have trdef $X=\omega_{0}$. Observe that for any compact subspace $Y$ of $X$ and each $n \geq 2$ there is a subset of $\left(\{1 / n\} \times E_{n}\right) \backslash Y$ homeomorphic to $\mathbb{I}^{n-1}$. Thus $\mathcal{K}_{0}$-trsur $X \geq \omega_{0}$. Then, by (4.2), it follows that $\mathcal{K}_{0}$-trsur $X=\omega_{0}$.

(i) By Propositions 4.5(ii) and 4.3 we have $M(\alpha)$-trind $X=\infty$. It is clear (see the hierarchy of absolute Borel classes) that $M(\alpha)$ - $\operatorname{trdef} X \leq \operatorname{trdef} X$ $=\omega_{0}$. Hence, by Remark 1.1(i), we get $M(\alpha)$-trdef $X=\omega_{0}$. Furthermore, since $Q_{\alpha} \in A(\alpha)$, by Propositions 3.3 and 3.4(ii) it follows that $E_{n}=$ $\pi_{n}^{-1}\left(Q_{\alpha}\right) \in A(\alpha)$. Then Proposition 3.1(i) yields $X \in A(\alpha)$. Hence $A(\alpha)$ trind $X=A(\alpha)$-trsur $X=-1$. 
(ii) Use Propositions 4.5(iii), 4.3 and Remark 1.1(ii) to get $A(\alpha)$-trind $X$ $=\infty$ and hence $A(\alpha)$-trsur $X=\omega_{0}$ by a similar argument to the one above. To prove that $M(\alpha)$-trind $X=M(\alpha)$-trdef $X=-1$, it suffices to show that $X \in M(\alpha)$. Since $P_{\alpha} \in M(\alpha)$, Propositions 3.3 and 3.4(ii) show that $E_{n}=\pi_{n}^{-1}\left(P_{\alpha}\right) \in M(\alpha)$. Hence $\mathbb{I}^{n} \backslash E_{n} \in A(\alpha)$ and $\bigcup_{n=2}^{\infty}\left(\mathbb{I}^{n} \backslash E_{n}\right) \in A(\alpha)$. Therefore, $X=Y \backslash \bigcup_{n=2}^{\infty}\left(\mathbb{I}^{n} \backslash E_{n}\right) \in M(\alpha)$.

(iii) Use Proposition 4.5(iv) to get $M(\alpha)$-trind $X=A(\alpha)$-trind $X=\infty$ and $M(\alpha)$-trdef $X=A(\alpha)$-trsur $X=\omega_{0}$ as above. Since $D_{\alpha} \in A(\alpha+1) \cap$ $M(\alpha+1)$, an argument similar to (ii) shows that $X \in A(\alpha+1) \cap M(\alpha+1)$ and hence $A(\alpha) \cap M(\alpha)$-trind $X=-1$.

(iv) By Proposition 4.5(v), $E_{n} \cap \bigcap_{i=1}^{m} L_{i}^{n} \notin \mathcal{A B}$. Hence Proposition 4.3 yields $\mathcal{A B}$-trind $X=\infty$. The proposition is proved.

Proof of Theorem 1.1. Let $\pi_{n}: \mathbb{I}^{n} \rightarrow \mathbb{I}$ be the projection onto the $n$th factor. For each ordinal $\alpha$ with $1 \leq \alpha<\omega_{1}$ we define

$$
\begin{aligned}
& X_{\alpha}=\left(\{0\} \times \mathbb{I}^{\infty}\right) \cup\left(\bigcup_{n=2}^{\infty}\{1 / n\} \times \pi_{n}^{-1}\left(P_{\alpha}\right)\right), \\
& Y_{\alpha}=\left(\{0\} \times \mathbb{I}^{\infty}\right) \cup\left(\bigcup_{n=2}^{\infty}\{1 / n\} \times \pi_{n}^{-1}\left(Q_{\alpha}\right)\right), \\
& Z_{\alpha}=\left(\{0\} \times \mathbb{I}^{\infty}\right) \cup\left(\bigcup_{n=2}^{\infty}\{1 / n\} \times \pi_{n}^{-1}\left(D_{\alpha}\right)\right) .
\end{aligned}
$$

It follows from Proposition 4.6 that $X_{\alpha}, Y_{\alpha}$ and $Z_{\alpha}$ satisfy conditions (i)-(iv). The second part of Theorem 1.1 is a direct consequence of the following facts.

Lemma 4.2. Let $X$ be a space with either $A(\alpha)$-trind $X=-1$ and $M(\alpha)$ trind $X \leq \mu$, or $A(\alpha)$-trind $X=\mu$ and $M(\alpha)$-trind $X=-1$, where $\mu$ is an ordinal or the integer -1 . Then $A(\alpha) \cap M(\alpha)$-trind $X \leq \mu$.

Proof. We consider only the case $A(\alpha)$-trind $X=-1$ and $M(\alpha)$-trind $X$ $\leq \mu$. We apply induction on $\mu \geq-1$. If $\mu=-1$ then $X \in A(\alpha) \cap M(\alpha)$. Hence $A(\alpha) \cap M(\alpha)$-trind $X=-1$. Thus the assertion is valid for $\mu=-1$. Assume that it holds for $\mu<\gamma \geq 0$. Let now $\mu=\gamma$. For each $x \in X$ and each neighborhood $U$ of $x$ there is a neighborhood $V \subset U$ of $x$ such that $M(\alpha)$-trind $\operatorname{Bd} V<\gamma$. Note that $A(\alpha)$-trind $\operatorname{Bd} V=-1$. Hence by the inductive assumption, we have $A(\alpha) \cap M(\alpha)$-trind $\mathrm{Bd} V<\gamma$. Therefore $A(\alpha) \cap M(\alpha)$-trind $X \leq \gamma$. The lemma is proved.

Proposition 4.7. Let $X$ be a space such that $A(\alpha)$-trind $X \leq \mu_{1}$ and $M(\alpha)$-trind $X \leq \mu_{2}$, where $\mu_{1}$ and $\mu_{2}$ are ordinals. Then 
$A(\alpha) \cap M(\alpha)$-trind $X$

$$
\leq \begin{cases}\mu_{1}+n\left(\mu_{2}\right)+1=\mu_{2}+n\left(\mu_{1}\right)+1 & \text { if } \lambda\left(\mu_{1}\right)=\lambda\left(\mu_{2}\right), \\ \mu_{1} & \text { if } \lambda\left(\mu_{1}\right)>\lambda\left(\mu_{2}\right), \\ \mu_{2} & \text { if } \lambda\left(\mu_{2}\right)>\lambda\left(\mu_{1}\right) .\end{cases}
$$

Proof. We apply induction on $\nu=\max \left\{\mu_{1}, \mu_{2}\right\} \geq 0$. If $\nu=0$ then $\mu_{1}=\mu_{2}=0$. For each $x \in X$ and each neighborhood $U$ of $x$ there is a neighborhood $V \subset U$ of $x$ such that $A(\alpha)$-trind $\mathrm{Bd} V=-1$. Observe that $M(\alpha)$-trind $\mathrm{Bd} V \leq 0$. Lemma 4.2 implies that $A(\alpha) \cap M(\alpha)$-trind $\mathrm{Bd} V \leq 0$. Hence $A(\alpha) \cap M(\alpha)$-trind $X \leq 1$, and the assertion is valid for $\nu=0$.

Suppose $\nu>0$ and the assertion holds for every $\gamma<\nu$.

CASE 1: $\lambda\left(\mu_{1}\right)>\lambda\left(\mu_{2}\right)$. Then $\nu=\mu_{1}$. For each $x \in X$ and each neighborhood $U$ of $x$ there is a neighborhood $V \subset U$ of $x$ such that $A(\alpha)$ trind $\mathrm{Bd} V<\mu_{1}=\nu$. Since $M(\alpha)$-trind $\mathrm{Bd} V \leq \mu_{2}<\lambda\left(\mu_{1}\right) \leq \mu_{1}=\nu$, by the inductive assumption we have $A(\alpha) \cap M(\alpha)$-trind $\mathrm{Bd} V<\mu_{1}$. Hence $A(\alpha) \cap M(\alpha)$-trind $X \leq \mu_{1}$.

Similarly the assertion is valid for $\lambda\left(\mu_{1}\right)>\lambda\left(\mu_{2}\right)$.

CASE 2: $\lambda\left(\mu_{1}\right)=\lambda\left(\mu_{2}\right)$ and $\mu_{1}>\mu_{2}$. Then $\nu=\mu_{1}$. For each $x \in X$ and each neighborhood $U$ of $x$ there is a neighborhood $V \subset U$ of $x$ such that $A(\alpha)$-trind $\mathrm{Bd} V<\mu_{1}$. The inductive assumption yields

$$
\begin{aligned}
A(\alpha) \cap M(\alpha) \text {-trind } \mathrm{Bd} V & \leq \mu_{1}-1+n\left(\mu_{2}\right)+1=\mu_{1}+n\left(\mu_{2}\right) \\
& <\mu_{1}+n\left(\mu_{2}\right)+1 .
\end{aligned}
$$

Hence $A(\alpha) \cap M(\alpha)$-trind $X \leq \mu_{1}+n\left(\mu_{2}\right)+1$. Analogously the assertion is valid for $\mu_{2}>\mu_{1}$.

CASE 3: $\mu_{1}=\mu_{2}=\mu$. If $\mu$ is not a limit ordinal, then for each $x \in X$ and each neighborhood $U$ of $x$ there is a neighborhood $V \subset U$ of $x$ such that $M(\alpha)$-trind $\operatorname{Bd} V \leq \mu-1$. By Case 2, we have

$$
A(\alpha) \cap M(\alpha) \text {-trindBd } V \leq \mu+n(\mu-1)+1=\mu+n(\mu) .
$$

So $A(\alpha) \cap M(\alpha)$-trind $X \leq \mu_{1}+n(\mu)+1$. If $\mu$ is a limit ordinal, then for each $x \in X$ and each neighborhood $U$ of $x$ there is a neighborhood $V \subset U$ of $x$ such that $M(\alpha)$-trind $\mathrm{Bd} V<\mu$. By Case 1 , we have $A(\alpha) \cap M(\alpha)$ trind $\mathrm{Bd} V \leq \mu$. Hence $A(\alpha) \cap M(\alpha)$-trind $X \leq \mu+1$. This completes the proof.

We do not know of any example of a space $X$ such that $\mathcal{C}_{0^{-}}$-ind $X=\mathcal{S}_{0^{-}}$ ind $X=0$ and $\mathcal{C}_{0} \cap \mathcal{S}_{0}$-ind $X=1$.

Proof of Theorem 1.2. Let $F$ be a Bernstein set in $\mathbb{I}$. Set $X=\left(\{0\} \times \mathbb{I}^{\infty}\right)$ $\cup\left(\bigcup_{n=2}^{\infty}\{1 / n\} \times \pi_{n}^{-1}(F)\right)$. Then Proposition 4.6 and the hierarchy of absolute Borel classes show that $X$ is as desired. 
Remark 4.2. By Proposition 4.6 the space $X$ from (4.1), where $E_{n}=$ $\left\{\left(x_{i}\right) \in \mathbb{I}^{n}: 0<x_{n} \leq 1\right\}$ for each $n \geq 2$, has $\operatorname{trcmp} X=\infty$ and $\operatorname{trdef} X$ $=\mathcal{K}_{0}$-trsur $X=\omega_{0}$. Evidently, $A(1) \cap M(1)$-trind $X=-1$.

Recall ([3, Theorem 7.1.6]) that for any space $X$, trind $X<\omega_{1}$ or trind $X=\infty$. In [6] R. Pol showed that for each ordinal $\beta<\omega_{1}$ there exists a separable completely metrizable $\sigma$-compact space $R_{\beta}$ with $\operatorname{trcmp} R_{\beta}=\beta$. Notice that $\mathcal{C}_{0}$-trind $R_{\beta}=\mathcal{S}_{0}$-trind $R_{\beta}=-1$. So it is natural to pose

Problem 4.1. Do there exist for each ordinal $1 \leq \alpha<\omega_{1}$ and each ordinal $0 \leq \beta<\omega_{1}$ spaces $X_{\alpha, \beta}, Y_{\alpha, \beta}$ such that $M(\alpha)$-trind $X_{\alpha, \beta}=A(\alpha)$ trind $Y_{\alpha, \beta}=\beta$ and $A(\alpha)$-trind $X_{\alpha, \beta}=M(\alpha)$-trind $Y_{\alpha, \beta}=-1$ ?

Remark 4.3. Let $\mathbb{Q}$ denote the space of rationals. In [6] R. Pol observed that using Aarts' argument in the proof of the equality $\operatorname{cmp}\left(\mathbb{I}^{n} \times \mathbb{Q}\right)=n([1])$ one can show that $\operatorname{trcmp}(X \times \mathbb{Q})=\alpha$ for any $X \in \mathcal{K}_{0}$ with trind $X=\alpha$. We can add that even $M(1)$-trind $(X \times \mathbb{Q})=M(1)$-trdef $(X \times \mathbb{Q})=\alpha$. (Evidently $A(1)$-trind $(X \times \mathbb{Q})=-1$.) Recall $([3])$ that for any $\alpha<\omega_{1}$ there is a space $X_{\alpha} \in \mathcal{K}_{0}$ such that trind $X_{\alpha}=\alpha$.

Acknowledgements. The results of this paper were obtained during a visit of the first author to Shimane University (Japan) supported by the Japan Society for Promotion of Science and the Royal Swedish Academy of Sciences. He thanks the Department of Mathematics of Shimane University for hosting him.

The authors would like to thank the referee for his/her suggestions.

\section{References}

[1] J. M. Aarts and T. Nishiura, Dimension and Extensions, North-Holland, Amsterdam, 1993.

[2] M. G. Charalambous, On transfinite inductive dimension and deficiency modulo a class $\mathcal{P}$, Topology Appl. 81 (1997) 123-135.

[3] R. Engelking, Theory of Dimensions, Finite and Infinite, Heldermann, Lemgo, 1995.

[4] A. Lelek, Dimension and mappings of spaces with finite deficiency, Colloq. Math. 12 (1964), 221-227.

[5] E. Pol, The Baire-category method in some compact extension problems, Pacific J. Math. 122 (1986), 197-210.

[6] -, On transfinite inductive compactness degree, Colloq. Math. 53 (1987), 57-61.

[7] L. R. Rubin, R. M. Schori and J. J. Walsh, New dimension-theory techniques for constructing infinite-dimensional examples, General Topology Appl. 10 (1979), 93102. 
[8] S. M. Srivastava, A Course on Borel Sets, Springer, New York, 1998.

Vitalij Chatyrko

Department of Mathematics

Linköping University

58183 Linköping, Sweden

E-mail:vitja@mai.liu.se
Yasunao Hattori Department of Mathematics Shimane University Matsue, 690-8504 Japan E-mail: hattori@riko.shimane-u.ac.jp

Received April 3, 2008;

received in final form May 30, 2008 